University of Nebraska - Lincoln DigitalCommons@University of Nebraska - Lincoln

$4-1986$

LINEARIZED FORM OF IMPLICIT TVD SCHEMES FOR THE MULTIDIMENSIONAL EULER AND NAVIER-STOKES EQUATIONS

Helen C. Yee

NASA Ames Research Center, yee@nas.nasa.gov

Follow this and additional works at: http://digitalcommons.unl.edu/nasapub

Yee, Helen C., "LINEARIZED FORM OF IMPLICIT TVD SCHEMES FOR THE MULTIDIMENSIONAL EULER AND NAVIER-STOKES EQUATIONS" (1986). NASA Publications. 249.

http://digitalcommons.unl.edu/nasapub/249

This Article is brought to you for free and open access by the National Aeronautics and Space Administration at DigitalCommons@University of Nebraska - Lincoln. It has been accepted for inclusion in NASA Publications by an authorized administrator of DigitalCommons@University of Nebraska - Lincoln. 


\title{
LINEARIZED FORM OF IMPLICIT TVD SCHEMES FOR THE MULTIDIMENSIONAL EULER AND NAVIER-STOKES EQUATIONS
}

\author{
H. C. YEE \\ Computational Fluid Dynamics Branch. MS 202A-1. NASA Ames Research Center. \\ Motfeu Field. CA 94035. LI.S.A.
}

\begin{abstract}
Linearized alternating direction inplicit (ADI) forms of a class of total variation diminishing (TVD) schemes for the Euler and Navier-Stokes equations have been developed. These schemes are based on the second-order-accurate TVD schemes for hyperbolic conservation laws developed by Harten[1.2]. They have the property of not generating spurious oscillations across shocks and contact discontinuities. In general, shocks can be captured within 1-2 grid points. These schemes are relatively simple to understand and easy to implement into a new or existing computer code. One can modify a standard three-point central-difference code by simply changing the conventional numerical dissipation term into the one designed for the TVD scheme. For steady-state applications. the only difference in computation is that the current schemes require a more elaborate dissipation term for the explicit operator: no extra computation is required for the inplicit operator. Numerical experiments with the proposed algorithms on a variety of steady-state airfoil problems illustrate the versatility of the schemes.
\end{abstract}

\section{INTRODUCTION}

Harten's method of constructing high-resolution TVD schemes involves starting with a firstorder TVD scheme and applying it to a modified flux. The modified flux is chosen so that the scheme is second order at regions of smoothness and first order at points of extrema. This technique is sometimes referred to as the modified flux approach. Although the scheme is an upwind scheme, it is written in a symmetric form; i.e. central difference plus a "smart" numerical dissipation term. This symmetric form is especially advantageous for systems of higher than one dimension. It results in less storage and a smaller operation count than its upwind form[3]. The modified flux approach is relatively simple to understand and easy to implement into a new or existing computer code. One can modify a standard three-point centraldifference code by simply changing the conventional numerical dissipation term into the one designed for the TVD scheme. However, for non-Cartesian grids. care must be taken to preserve freestream. A formulation closer to finite volume would be more desirable.

In [4]. a preliminary study was done on an implicit TVD scheme for a two-dimensional gas dynamics problem in a Cartesian grid. It was found that further improvement in computational efficiency and convergence rate is required for practical application.

The objective of this paper is to formulate linearized forms and to develop the various solution strategies for the implicit TVD schemes for two-dimensional Euler and Navier-Stokes equations. In particular, the study of more efficient formulations for two-dimensional steadystate applications is emphasized. Numerical experiments with some of the 1984 AGARD Fluid Dynamics Panel Working Group 07 airfoil test cases[5] are included.

\section{EXPLICIT TVD SCHEMES}

First-order explicit TVD scheme

Consider the scalar hyperbolic conservation law

$$
\frac{\partial u}{\partial t}+\frac{\partial f(u)}{\partial x}=0
$$

where $f$ is the flux and $a(u)=\partial f / \partial u$ is the characteristic speed. Let $u_{\prime \prime}^{\prime \prime}$ be the numerical solution of (2.1) at $x=j \Delta x$ and $t=n \Delta t$, with $\Delta x$ the spatial mesh size and $\Delta t$ the time step. 
A general three-point explicit-difference scheme in conservation form can be written as

$$
u_{j}^{n+1}=u_{j}^{n}-\lambda\left(h_{j+1}^{n}=-h_{j-12}^{n}\right),
$$

where $h_{j+1 / 2}^{n}=h\left(u_{j}^{n}, u_{j+1}^{n}\right)$, and $\lambda=\Delta t / \Delta x$. Here, $h$, commonly called a numerical flux function, is required to be consistent with the conservation law in the following sense:

$$
h\left(u_{j}, u_{j}\right)=f\left(u_{j}\right)
$$

Consider a numerical scheme with a numerical flux function of the following form:

$$
h_{j+1 / 2}=\frac{1}{2}\left[f_{j}+f_{j+1}-\psi\left(a_{j+1: 2}\right) \Delta_{j+1,2} u\right],
$$

where $f_{j}=f\left(u_{j}\right), \Delta_{j+1 / 2} u=u_{j+1}-u_{j}$ and

$$
a_{j+1 / 2}= \begin{cases}\left(f_{j+1}-f_{j}\right) / \Delta_{j+1: 2} u & \Delta_{j+1: 2} u \neq 0 . \\ a\left(u_{j}\right) & \Delta_{j+1: 2} u=0 .\end{cases}
$$

Here $\psi$ is a function of $a_{j+1 / 2}$ and $\lambda$. The function $\psi$ is sometimes referred to as the coefficient of numerical viscosity. A scheme with a numerical flux of the form (2.4) is the first-order accurate upwind scheme[6,7]

$$
u_{j}^{n+1}=u_{j}^{n}-\frac{\lambda}{2}\left[1-\operatorname{sgn}\left(a_{j+1 / 2}^{n}\right)\right]\left(f_{j+1}^{n}-f_{j}^{n}\right)-\frac{\lambda}{2}\left[1+\operatorname{sgn}\left(a_{j-1 / 2}^{n}\right)\right]\left(f_{j}^{n}-f_{j-1}^{n}\right)
$$

If we define $a_{j+1 / 2}$ as $(2.4 \mathrm{~b})$, then (2.5) can be written as

$$
u_{j}^{n+1}=u_{j}^{n}-\frac{\lambda}{2}\left[f_{j+1}^{n}-f_{j-1}^{n}-\left|a_{j+1 / 2}^{n}\right| \Delta_{j-1 ; 2} u^{n}+\left|a_{j-1 / 2}^{n}\right| \Delta_{j-1,2} u^{n}\right]
$$

Here, the numerical flux function is

$$
h_{j+1 / 2}=\frac{1}{2}\left[f_{j}+f_{j+1}-\left|a_{j+1 ; 2}\right| \Delta_{j+1 / 2} u\right]
$$

with

$$
\psi\left(a_{j+1 / 2}\right)=\left|a_{j+1}\right|
$$

This scheme is sometimes known as the Huang Scheme[6], or the Roe Scheme[7]. It is well known that (2.5) and (2.6) are not consistent with an entropy inequality, and the scheme might converge to a nonphysical solution. A slight modification of the numerical viscosity term

$$
\psi(z)= \begin{cases}|z| & |z| \geq \epsilon, \\ \left(z^{2}+\epsilon^{2}\right) / 2 \epsilon & |z|<\epsilon\end{cases}
$$

can remedy the entropy violating problem[1], where $\epsilon>0$ is a parameter. A formula for $\epsilon$ can be found in Ref. [8]. The $\psi(z)$ in (2.7) is a continuously differentiable positive approximation to $|z|$ in $(2.6 \mathrm{c})$. The notation of the numerical flux function $(2.4 \mathrm{a})$ will be used heavily for the rest of the paper.

If one defines

$$
C^{ \pm}(z)=\frac{1}{2}[\psi(z) \pm z]
$$


then Eq. (2.2) together with (2.4) can be written as

$$
u_{i}^{n+1}=u_{i}^{\prime \prime}+\lambda C^{-}\left(a_{i+1,2}^{\prime \prime}\right) \Delta_{i+1} u^{\prime \prime}-\lambda C^{-}\left(a_{1-1,1}^{\prime \prime}\right) \Delta_{j-1} u^{\prime \prime} .
$$

The total variation of a mesh function $u^{\prime \prime}$ is defined to be

$$
\operatorname{TV}\left(u^{\prime \prime}\right)=\sum_{j=-x}^{x}\left|u_{j+1}^{\prime \prime}-u_{j}^{\prime \prime}\right|=\sum_{j=-\infty}^{x}\left|\Delta_{j+1} \geq u^{\prime \prime}\right|
$$

The numerical scheme (2.2) for an initial-value problem of (2.1) is said to be TVD if

$$
\operatorname{TV}\left(u^{\prime \prime+1}\right) \leq \operatorname{TV}\left(u^{\prime \prime}\right)
$$

It can be shown that sufficient conditions [1] for (2.2), together with (2.4), to be a TVD scheme are

$$
\begin{aligned}
\lambda C^{-}\left(a_{j+1}\right) & \geq 0, \\
\lambda C^{+}\left(a_{j+1}\right) & \geq 0, \\
\lambda\left[C^{-}\left(a_{j+1 / 2}\right)+C^{+}\left(a_{i+1,2}\right)\right] & \leq 1 .
\end{aligned}
$$

Applying the above conditions to Eq. (2.6), it can be easily shown that (2.6) is a TVD scheme. Therefore, for the scheme (2.2) with a general $\psi$ in $(2.4 \mathrm{a})$ [other than (2.6c)] to be TVD. we have to pick $\psi$ such that $(2.12)$ is satisfied.

\section{Second-order explicit TVD scheme}

In [1], Harten converted the first-order scheme (2.2) into a second-order TVD scheme by applying it to a modified flux $\bar{f}(u)=[f(u)+g(u)]$. The new numerical flux function $\bar{h}_{j+1}$, depends on $(f+g)$ instead of $f$ alone, the coefficient of the numerical viscosity term $\psi$ is a function of a modified characteristic speed $(a+\gamma)$. and $\bar{h}_{1+1}$, can be written as

$$
\bar{h}_{j+1 / 2}=\frac{1}{2}\left[\bar{f}_{j}+\tilde{f}_{i+1}-\psi\left(a_{i+1,2}+\gamma_{1+1 \cdot 2}\right) \Delta_{1-1.2} u t\right.
$$

where $\bar{f}_{j}=f_{j}+g_{i}$ and $\gamma_{1+1,2}$ is defined almost the same way as $a_{i+1}$ except that it is a function of the $g$,'s instead of the $f_{j}$ 's. It can be expressed as

$$
\gamma_{j+1 / 2}= \begin{cases}\left(g_{j+1}-g_{j}\right) / \Delta_{i+1} 2 u & \Delta_{i+12} u \neq 0 \\ 0 & \Delta_{i+12} \|=0\end{cases}
$$

The requirements on $g$ are (i) the function $g$ should have a bounded $\gamma$ in $(2.13 b)$ so that the scheme (2.2) together with (2.13) is TVD with respect to the modified flux $(f+g)$. and (ii) the modified scheme should be second-order accurate (except at points of extrema). In [1.2], Harten devised a recipe for $g$ that satisfies the above two requirements. We will use this particular form of $g$ for the discussion here. It can be written as

$$
\begin{aligned}
g_{j} & =S \cdot \max \left[0, \min \left(\sigma_{j+1},\left|\Delta_{j+1} \geq u\right| . S \cdot \sigma_{1-1,2} \Delta_{l-1}, u\right)\right] . \\
S & =\operatorname{sgn}\left(\Delta_{i+1,2} u\right),
\end{aligned}
$$

with $\sigma_{j+1,2}=\sigma\left(a_{j+12}\right)$, and we choose

$$
\sigma(z)=\frac{1}{2}[\psi(z)-\lambda=2] \geq 0
$$


for time-accurate calculations. With this choice of $\sigma(z)$, the scheme (2.2) together with (2.13) is second-order accurate in both time and space (except at points of extrema); see [1,2]. Also. with this choice of the $g$ function, the second-order TVD scheme will automatically switch itself to first-order at points of extrema. This is one of the vehicles to prevent spurious oscillation near a shock. Other more general forms for $g$ can be obtained following the line of argument of Sweby[9] and Roe[10]. The function $g_{j}$ is sometimes referred to as the "minmod" function of the argument indicated. It is a form of the so-called "limiter" for the control of unwanted oscillations in numerical schemes. A more uniform second-order nonoscillatory scheme is under development by Harten and Osher[11]. Their new scheme requires a higher operations count than the current one.

The second-order TVD scheme can be written as

$$
u_{j}^{n+1}=u_{j}^{n}+\lambda C^{-}\left(\tilde{a}_{j+1: 2}^{n}\right) \Delta_{j+1} \geq u^{n}-\lambda C^{+}\left(\tilde{a}_{j-1}^{n}\right) \Delta_{j-1}: 2 u^{n}
$$

with $\tilde{a}_{j+1 / 2}^{n}=a_{j+1 / 2}^{n}+\gamma_{j+12}^{n}$. As a side remark, with the choice of $g_{j}$ above, $\operatorname{sgn}\left(\bar{a}_{j+12}^{n}\right)=$ $\operatorname{sgn}\left(a_{j+1 / 2}^{n}\right)$.

Another interesting observation is that Eq. (2.13) together with (2.6c) can be rewritten as

$$
u_{j}^{n+1}=u_{j}^{n}-\frac{\lambda}{2}\left[1-\operatorname{sgn}\left(\tilde{a}_{j+1 / 2}^{n}\right)\right]\left(\tilde{f}_{j+1}^{n}-\tilde{f}_{j}^{n}\right)-\frac{\lambda}{2}\left[1+\operatorname{sgn}\left(\tilde{a}_{j-1 \cdot 2}^{n}\right)\right]\left(\tilde{f}_{j}^{n}-\tilde{f}_{j-1}^{n}\right) .
$$

This is a straightforward extension of Huang or Roe's entropy-violating first-order upwind scheme to second-order accuracy. The scheme looks identical to their first-order scheme (2.5) except the arguments $a$ 's and $f$ 's are different. Here

$$
\tilde{h}_{j+1}=\frac{1}{2}\left[1-\operatorname{sgn}\left(\tilde{a}_{j+1}\right)\right]\left(\tilde{f}_{j+1}-\bar{f}_{j}\right)-\tilde{f}_{j} .
$$

Equation (2.16) is identical to (2.13a) if one defines $\psi(z)=|z|$ in $(2.13)$. Since $\operatorname{sgn}\left(\tilde{a}_{j+1}^{n} z^{\prime}\right)=$ $\operatorname{sgn}\left(a_{j+1 / 2}^{n}\right)$, Eq. $(2.15)$ and $(2.16)$ are also equal to

$$
u_{j}^{n+1}=u_{j}^{n}-\frac{\lambda}{2}\left[1-\operatorname{sgn}\left(a_{j+1 / 2}^{n}\right)\right]\left(\tilde{f}_{j+1}^{n}-\tilde{f}_{j}^{n}\right)-\frac{\lambda}{2}\left[1+\operatorname{sgn}\left(a_{j-1}^{n}\right)\right]\left(\tilde{f}_{j}^{n}-\tilde{f}_{j-1}^{n}\right) .
$$

with

$$
\tilde{h}_{j+1,2}=\frac{1}{2}\left[1-\operatorname{sgn}\left(a_{j+1: 2}\right)\right]\left(\tilde{f}_{j+1}-\bar{f}_{j}\right)+\tilde{f}_{j}
$$

With this formulation, one does not have to calculate $\gamma_{j+1,2}$ at all.

For the numerical flux (2.16), the $g$ function of $(2.13 \mathrm{c})$ can be defined in a slightly different form:

$$
\begin{aligned}
g_{j} & =S \cdot \max \left[0, \min \left(\bar{\sigma}_{j+1 / 2}\left|\Delta_{j+1}, 2 f\right|, S \cdot \bar{\sigma}_{j-1,2} \Delta_{j-1,2} f\right)\right], \\
S & =\operatorname{sgn}\left(\Delta_{j+1 / 2} f\right),
\end{aligned}
$$

with $\bar{\sigma}_{j+1 / 2}=\bar{\sigma}\left(a_{j+1 / 2}\right)$ and

$$
\bar{\sigma}(z)=\frac{1}{2}[\operatorname{sgn}(z)-\lambda z]
$$

Here the identities $\operatorname{sgn}\left(\Delta_{j+1}, 2\right) \operatorname{sgn}\left(a_{j+1,2}\right)=\operatorname{sgn}\left(u_{j+1}-u_{j}\right)$ and $\left|\Delta_{-12} f\right|=\left|a_{j+1}\right|\left|u_{j+1}-u_{j}\right|$ are used. The above limiter $g_{j}$ of (2.19) can be considered as a flux limiter since the flux $f$ is limited. Equations $(2.13 c, d)$ are preferred over (2.19) for its straightforward extension to system cases because $u$ appears rather than $f$. 
First-order implicit TVD scheme

Now consider a one-parameter family of three-point conservative schemes of the form

$$
u_{j}^{n+1}+\lambda \theta\left(h_{j+1 / 2}^{n+1}-h_{j-1 / 2}^{n+1}\right)=u_{j}^{n}-\lambda(1-\theta)\left(h_{j+1 / 2}^{n}-h_{j-1 / 2}^{n}\right),
$$

where $0 \leq \theta \leq 1$ is a parameter, $h_{j+1 / 2}^{n}=h\left(u_{j}^{n}, u_{j+1}^{n}\right), h_{j+1}^{n+1}=h\left(u_{j}^{n+1}, u_{j+1}^{n+1}\right)$, and $h\left(u_{j}, u_{j+1}\right)$ is the numerical flux (2.4). This one-parameter family of schemes contains implicit as well as explicit schemes. When $\theta=0,(3.1)$ reduces to (2.2), the explicit method. When $\theta \neq 0,(3.1)$ is an implicit scheme. For example, if $\theta=1 / 2$, the time differencing is the trapezoidal formula. and if $\theta=1$, the time differencing is the backward Euler method. To simplify the notation. rewrite (3.1) as

$$
L \cdot u^{n+1}=R \cdot u^{n},
$$

where $L$ and $R$ are the following finite-difference operators:

$$
\begin{aligned}
& (L \cdot u)_{j}=u_{j}+\lambda \theta\left(h_{j+1 / 2}-h_{j-1 / 2}\right), \\
& (R \cdot u)_{j}=u_{j}-\lambda(1-\theta)\left(h_{j+1 / 2}-h_{j-1,2}\right) .
\end{aligned}
$$

Sufficient conditions for ( 3.1$)$ to be a TVD scheme are that

$$
\operatorname{TV}\left(R \cdot u^{n}\right) \leq \operatorname{TV}\left(u^{n}\right)
$$

and

$$
\operatorname{TV}\left(L \cdot u^{n+1}\right) \geq \operatorname{TV}\left(u^{n+1}\right) .
$$

A sufficient condition for (3.4) is the CFL-like restriction

$$
\left|\lambda a_{j+1 / 2}\right| \leq \lambda \psi\left(a_{j+1 / 2}\right) \leq \frac{1}{1-\theta},
$$

where $a_{j+1 / 2}$ is defined in Eq. (2.5b). Therefore, for the scheme to be TVD, one has to pick $\psi\left(a_{j+1 / 2}\right)$ such that (3.5) is satisfied. For a detailed proof of Eqs. (3.4) and (3.5), see [2]. Observe that the backward Euler implicit scheme, $\theta=1$ in $(3.1)$, is unconditionally TVD. while the trapezoidal formula, $\theta=1 / 2$ is TVD under the CFL-like restriction of 2 . The forward Euler explicit scheme, $\theta=0$ or Eq. (2.2), is TVD under the CFL restriction of 1 .

\section{Second-order implicit TVD scheme}

One can obtain a second-order-accurate implicit TVD scheme by replacing the numerical flux function $h$ of (3.1) with $\tilde{h}$ of Eqs. (2.13) or (2.18); i.e.

$$
u_{j}^{n+1}+\lambda \theta\left(\bar{h}_{j+1 / 2}^{n+1}-\bar{h}_{j-1 ; 2}^{n+1}\right)=u_{j}^{n}-\lambda(1-\theta)\left(\bar{h}_{j+1 / 2}^{n}-\bar{h}_{j-1 / 2}^{n}\right)
$$

However, $\sigma_{j+1 / 2}$ is different from (2.13d). Instead, choose

$$
\sigma(z)=\frac{1}{2} \psi(z)+\lambda\left(\theta-\frac{1}{2}\right) z^{\prime}
$$

for time-dependent calculations, or

$$
\sigma(z)=\frac{1}{2} \psi(z)
$$

for either steady-state or time-accurate calculations. 
For the first choice $(3.7 \mathrm{a})$, the scheme is second-order-accurate in space and time regardless of $\theta$. However. the steady-state solution depends on the time step. The second choice in (3.7b) makes the scheme second-order accurate in space but first-order accurate in time if $\theta=1$. This choice of $\sigma(z)$ ensures that the steady-state solution does not depend on the time step $\Delta t$. Secondorder in space and time can be achieved for $(3.7 b)$ if $\theta=1: 2$ is chosen. For example, an unconditionally TVD backward Euler scheme is of the form

$$
u_{1}^{\prime \prime-1}+\lambda\left(\bar{h}_{i+1}^{\prime \prime+1}-\bar{h}_{i-1}^{\prime \prime} 1_{2}\right)=u_{i}^{\prime \prime} .
$$

This is a highly nonlinear implicit scheme. An efficient procedure to solve this set of nonlinear equations is needed. The following focuses on linearized forms of the implicit scheme (3.6).

\section{Linearized nonconservalive implicit ( $L N I)$ form}

For steady-state calculations, the following linearized version of (3.6) in delta formulation $[4.12 \mid$ can be used:

$$
E_{1} d_{1-1}+E_{2} d_{j}+E_{3} d_{1-1}=-\lambda\left|\overline{h_{1-1}^{\prime \prime}}{ }_{2}-\overline{h_{1-1}^{\prime \prime}}\right|
$$

with

$$
\begin{aligned}
& E_{1}=-\lambda \theta\left(C_{i-1}^{+}\right)^{\prime \prime} . \\
& E_{2}=1+\lambda \theta\left[\left(C_{i-12}^{-1}\right)^{\prime \prime}+\left(C_{i-1}\right)^{n}\right] . \\
& E_{3}=-\lambda \theta\left(C_{i+12}^{+}\right)^{\prime \prime},
\end{aligned}
$$

where $d_{j}=u_{j}^{n-1}-u_{j}^{n} \cdot \bar{h}_{j+1}$, from (2.13) or (2.18), and

$$
\left(C^{=}\right)^{n}=\frac{1}{2}[\psi(a+\gamma) \pm(a+\gamma)]^{n} .
$$

One can obtain Eq. (3.9) by simply rewriting (3.6) in an upwind form so that the resulting

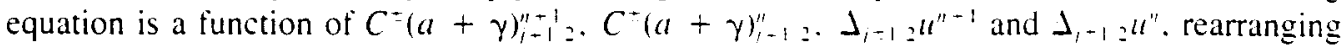
terms, and dropping the time index of the $C^{-}$from $(n+1)$ to $n$. Equation (3.9) will be referred to as the linearized nonconservative implicit (LNI) form.

Although (3.9) is formally a five-point scheme. the coefficient matrix associated with it is tridiagonal with a dominant diagonal. Another TVD linearized form can also be obtained by setting $\gamma=0$ in $(3.9 \mathrm{e})$; i.e., redefining $(3.9 \mathrm{e})$ by

$$
\left(C^{*}\right)^{n}=\frac{1}{2}|\psi(a) \pm a|^{\prime \prime} .
$$

Scheme (3.9a) together with (3.10) is spatially first-order accurate for the implicit operator and spatially second-order accurate for the explicit operator. It can be shown that $(3.9 \mathrm{a})$ together with (3.10) is still TVD. The LNI forms (3.9) and (3.10) are mainly useful for steady-state calculations, since the scheme is only conservative after the solution reaches steady state. i.e. if a steady state is attained the solution is the same as would have been obtained with a conservative scheme.

\section{Linearized conseriative implicit ( $L C l)$ form}

A linearized conservative implicit ( $\mathrm{LCl}$ ) form can be obtained by rewriting $12.13 a$ ) as

$$
\begin{aligned}
& h_{i+1}^{\prime \prime+1}=\frac{1}{2}\left[f_{i}^{n-1}+f_{i=1}^{n+1}\right] \\
& +\frac{1}{2}\left[\left(\frac{g_{1}+g_{1}-1}{\Delta_{1-1} u}\right) \Delta_{1-1} \geq u-\psi\left(a_{1-1}=+\gamma_{1-1},\right)_{1-1} \geq u\right]^{1-1} \text {. }
\end{aligned}
$$


and using a local Taylor expansion about $u^{\prime \prime}$ :

$$
f^{\prime \prime-1}-f^{\prime \prime}=u^{\prime \prime}\left(u^{\prime \prime-1}-u^{\prime \prime}\right)+O\left(\Delta t^{2}\right) \text {. }
$$

where $a=\partial f / \partial u$. Applying the first-order approximation of (3.12) and locally linearizing the coefficients of $\lambda_{1-1} u$ in the second and third terms on the right-hand-side by dropping the time index from $(n+1)$ to $n$. one gets the $\mathrm{LCl}$ form in delta formulation:

$$
\left.\bar{E}_{1} d_{1-1}+\bar{E}_{2} d_{1}+\bar{E}_{3} d_{1-1}=-\lambda \mid \bar{h}_{1-12}^{\prime \prime}-\bar{h}_{1-1}^{\prime \prime}\right]
$$

where

$$
\begin{aligned}
& \bar{E}_{1}=\frac{\lambda \theta}{2}\left[-a_{1-1}^{\prime \prime}+\beta_{,-1}^{\prime \prime}=-\psi\left((a+\gamma)_{1-1}^{\prime \prime}\right)\right] . \\
& \bar{E}_{z}=1+\frac{\lambda \theta}{2}\left[-\beta_{i+12}^{n}+\psi\left((a+\gamma)_{1-12}^{\prime \prime}\right)-\beta_{i-12}^{n}+\psi\left((a+\gamma)_{1-12}^{n}\right)\right] . \\
& \bar{E}_{:}=\frac{\lambda \theta}{2}\left[a_{1-1}^{n}+\beta_{i-12}^{n}-w\left((a+\gamma)_{1-1,2}^{\prime \prime}\right)\right] .
\end{aligned}
$$

and

$$
\beta_{i+12}^{n}=\left(\frac{g_{i}+g_{i-1}}{J_{1-12} \|}\right)^{n} .
$$

Again. this is a five-point scheme but with a tridiagonal coefficient matrix. An LCI form which is spatially first-order accurate can also be obtained for the implicit operator by simply setting $\gamma_{1-1}=\beta_{1-12}=0$ in $(3.13 \mathrm{~b})-(3.13 \mathrm{~d})$. These $\mathrm{LCl}$ forms preserve the conservative form of the differencing scheme; thus they are applicable to unsteady as well as steady-state calculations. The main drawback is that the resulting scheme may no longer be unconditionally TVD for $\theta=1$. There is no proof to show that the LCI form is still unconditionally TVD. Indeed, numerical experiments with the Burgers' equation show that the scheme (3.13) is not unconditionally TVD. This shortcoming of the LCI form appears to be disturbing at first. In practice. however. unconditional stability arising from linear stability analysis rarely carries over to nonlinear system cases with discontinuous solutions. Our main interest is a less restrictive time-step bound compared with explicit methods. Therefore, if the LCI form can offer a larger time-step bound with high accuracy, it should be sufficient for practical applications. As can be seen later. application of a variant of (3.1) for the Euler equation of gas dynamics in two dimensions shows that the $\mathrm{LCI}$ is a fairly useful tool for practical calculations. The method remains stable for fairly large CFL.

\section{FORMAL EXTENSION OF ALGORITHM FOR TWO-DIMENSIONAL SYSTEM OF HYPERBOLIC CONSERVATION LAWS}

Before going on to the next section, it should be emphasized that all the second-order TVD schemes are constructed so that no spurious oscillations are generated for one-dimensional nonlinear scalar hyperbolic conservation laws and constant-coefficient hyperbolic systems. None of the theory says anything about nonlinear systems or two-dimensional scalar hyperbolic conservation laws. Moreover. Goodman and Leveque[13] have obtained the following result: for a specific norm. a two-dimensional scalar approximation cannot be TVD and still be more than first-order accurate. Maybe what one needs is a different norm or a more relaxed detinition than the TVD property for one dimension. But in practice. it is straightforward to formally extend the scheme to one- or two-dimensional nonlinear hyperbolic systems. Surprisingly enough. numerical experiments with these schemes which are based on a one-dimensional concept applied 
to two-dimensional problems via local one-dimensional splitting show that these types of schemes do perform well for fairly complex shock structures[12,14].

Therefore, for the rest of the paper it is understood that the properties of all the schemes under discussion are for one-dimensional nonlinear scalar hyperbolic conservation laws and one-dimensional constant-coefficient hyperbolic systems. The schemes are then formally extended to one- or two-dimensional systems of conservation laws and are evaluated by numerical experiments.

Extension of the scalar TVD scheme to systems of conservation laws can be accomplished by defining at each point a "local" system of characteristic fields, and then applying the scheme to each of the $m$ scalar characteristic equations. Here $m$ is the dimension of the hyperbolic system. The formulation described here is valid for both two- and three-dimensional systems of conservation laws. Only the two-dimensional case will be described. For three-dimensional formulations, one only has to add an extra dimension and the corresponding numerical flux. Also, for simplicity of presentation, only conservation laws in Cartesian coordinates with equal spatial step size will be discussed. The variable step size or generalized coordinate formulations with application to airfoil calculations are reported in separate papers[15.16].

Consider a two-dimensional system of hyperbolic conservation laws

$$
\frac{\partial U}{\partial t}+\frac{\partial F(U)}{\partial x}+\frac{\partial G(U)}{\partial y}=0
$$

Here $U, F(U)$ and $G(U)$ are column vectors of $m$ components. Let $A=\partial F / \partial U$ and $B=$ $\partial G / \partial U$. Let the eigenvalues of $A$ be $\left(a_{x}^{1}, a_{x}^{2}, \ldots a_{x}^{m}\right)$ and the eigenvalues of $B$ be $\left(a_{x}^{1}, a_{y}^{2}\right.$. $\left.\ldots a_{y}^{m}\right)$. Denote $R_{x}$ and $R_{y}$ as the matrices whose columns are eigenvectors of $A$ and $B$, and denote $R_{x}^{-1}$ and $R_{y}^{-1}$ as the inverses of $R_{x}$ and $R_{y}$. In the case of the compressible Euler equation of gas dynamics

$$
U=\left[\begin{array}{c}
\rho \\
\rho u \\
\rho v \\
e
\end{array}\right] ; \quad F=\left[\begin{array}{c}
\rho u \\
\rho u^{2}+p \\
\rho u v \\
u(e+p)
\end{array}\right] ; \quad G=\left[\begin{array}{c}
\rho v \\
\rho u v \\
\rho v^{2}+p \\
v(e+p)
\end{array}\right] \text {. }
$$

The variables are the density $\rho$, the velocity components $u$ and $v$, and the pressure $p$. The total energy per unit volume, $e$, is related to $p$ by the equation of state for a perfect gas:

$$
p=(\gamma-1)\left[e-\frac{(\rho u)^{2}+(\rho v)^{2}}{2 \rho}\right]
$$

where $\gamma$ is the ratio of specific heats and should not be confused with the $\gamma_{1-1}$ in $(2.13 \mathrm{~b})$. The eigenvalues of $A$ are

$$
\left(a_{r}^{1}, a_{r}^{2}, a_{r}^{3}, a_{r}^{4}\right)=(u-c, u, u+c, u)
$$

where $c$ is the local speed of sound. The right eigenvectors of $A$ are given by

$$
R_{x}=\left[\begin{array}{cccc}
1 & 1 & 1 & 0 \\
u-c & u & u+c & 0 \\
v & v & v & 1 \\
H-u c & \left(u^{2}+v^{2}\right) / 2 & H+u c & v
\end{array}\right] .
$$

where

$$
H=\frac{c^{2}}{\gamma-1}+\frac{u^{2}+v^{2}}{2} .
$$


Similarly, the eigenvalues of $B$ are

$$
\left(a_{y}^{1}, a_{v}^{2}, a_{y}^{3}, a_{y}^{+}\right)=(v-c, v, v+c, v)
$$

The right eigenvectors of $B$ are given by

$$
R_{y}=\left[\begin{array}{cccc}
1 & 1 & 1 & 0 \\
u & u & u & 1 \\
v-c & v & v+c & 0 \\
H-v c & \left(u^{2}+v^{2}\right) / 2 & H+v c & u
\end{array}\right] .
$$

Let the grid spacing be denoted by $\Delta x$ and $\Delta y$ such that $x=j \Delta x$ and $y=k \Delta y$. Denote $U_{j+1 / 2 . k}$ as some symmetric average of $U_{j, k}$ and $U_{j+1, k}$ (for example, the arithmetic mean average or the Roe's average[7] for gas dynamics). Let $a_{j+1 / 2}^{l}, R_{j+1,2}, R_{j+1: 2}^{-1}$ denote the quantities $a_{x}^{l}$, $R_{x}, R_{x}^{-1}$ evaluated at $U_{j+1: 2 . k}$. Similarly, let $a_{k+1 / 2}^{\prime}, R_{k+1 / 2}, R_{k+1 / 2}^{-1}$ denote the quantities $a_{y}^{l}, R_{y}$, $R_{v}^{-1}$ evaluated at $U_{j, k+1 / 2}$.

Define

$$
\alpha_{j+1 / 2}=R_{j+1 / 2}^{-1}\left(U_{j+1, k}-U_{j, k}\right)
$$

as the difference of the characteristic variables in the locally $x$ direction, and define

$$
\alpha_{k+1 / 2}=R_{k+1 / 2}^{-1}\left(U_{j, k+1}-U_{j, k}\right)
$$

as the difference of the characteristic variables in the locally $y$ direction.

With the above notation, a one-parameter family of TVD schemes (3.6) together with $(2.13 a-c)$ and $(3.7 b)$ in two dimensions can be written as

$$
\begin{aligned}
& U_{j, k}^{n+1}+\lambda^{r} \theta\left(\tilde{F}_{j+1,2 . k}^{n+1}-\dot{F}_{j-1 \cdot 2, k}^{n+1}\right)+\lambda^{r} \theta\left(\tilde{G}_{j, k+1,2}^{n+1}-\tilde{G}_{j, k-1 / 2}^{n+1}\right) \\
& =U_{j . k}^{n}-\lambda^{x}(1-\theta)\left(\bar{F}_{j+1 / 2 . k}^{n}-\bar{F}_{j-1 / 2 . k}^{n}\right)-\lambda^{y}(1-\theta)\left(\bar{G}_{j, k+1 / 2}^{n}-\bar{G}_{j . k-1,2}^{n}\right),
\end{aligned}
$$

with $\lambda^{r}=\Delta t / \Delta x, \lambda^{y}=\Delta t / \Delta y$, and the numerical flux function $\bar{F}_{j+1 \cdot 2 . k}$ defined as

$$
\tilde{F}_{j+1 / 2 . k}=\frac{1}{2}\left[F_{j, k}+F_{j+1, k}+R_{j+1 / 2} \Phi_{j+1.2}\right] \text {. }
$$

where the elements of the $\Phi_{j+1 / 2}$ denoted by $\phi_{j+1 / 2}^{\prime}, l=1, \ldots m$ are

$$
\begin{aligned}
\phi_{j+1 / 2}^{l} & =g_{j}^{l}+g_{j+1}^{l}-\psi\left(a_{j+1 / 2}^{l}+\gamma_{j+1 / 2}^{l}\right) \alpha_{j+1,2}^{l}, \\
g_{j}^{l} & =S \cdot \max \left[0, \min \left(\sigma_{j+1 / 2}^{l}\left|\alpha_{j+1 / 2}^{l}\right|, S \cdot \sigma_{j-1 / 2}^{l} \alpha_{j-1 / 2}^{l}\right)\right], \\
S & =\operatorname{sgn}\left(\alpha_{j+1 / 2}^{l}\right),
\end{aligned}
$$

with $\psi(z)$ defined in (2.7); $\sigma_{j+1 / 2}^{\prime}$ is $(3.7 \mathrm{a})$ or (3.7b) with $z=a_{j+1}^{l}$, , and

$$
\gamma_{j+1 / 2}^{\prime}= \begin{cases}\left(g_{j+1}^{l}-g_{j}^{l}\right) / \alpha_{j+1 / 2}^{l} & \alpha_{j+1: 2}^{l} \neq 0 \\ 0 & \alpha_{j+1: 2}^{l}=0\end{cases}
$$

where $\alpha_{j+1,2}^{i}$ are the elements of (4.3a). The numerical flux $\bar{G}_{j . k+1}$, can be defined in a similar manner. Here, the formula (4.4) is only valid for $\sigma(z)=\psi(z) / 2$. If one uses $\sigma(z)=\psi / 2-$ $\lambda(\theta-1 / 2) z^{2}$, then the effect of the cross derivative $U_{x t}$ is neglected in formulation (4.4).

Alternate form for the numerical fluxes

Extension of the scalar TVD scheme to nonlinear system cases is not unique. Take, for example, the case where the numerical flux $\bar{h}_{j+1}$ in $(2.13)$ is identical to $\bar{h}_{j+1}$ in $(2.16)$ if 
one sets $\psi(z)=|z|$ in (2.13). The corresponding numerical fluxes for the system case has a different form depending on which of the scalars $\bar{h}_{1-1}:$ one started with. If one started with (2.16). $\dot{F}_{,+1, k}$ can be of the form

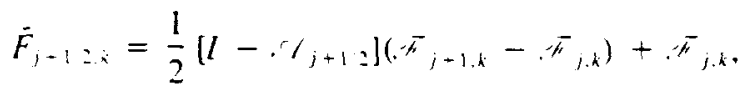

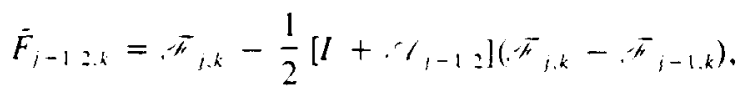

where

$$
\begin{aligned}
r /_{j+1 / 2} & =R_{j+1,2}^{-1} \Lambda_{,+1,2} R_{j+1 / 2}, \\
\Lambda & =\operatorname{diag}\left[\operatorname{sgn}\left(a_{j+1,2}\right)\right]
\end{aligned}
$$

and

$$
\bar{\pi}_{j . k}=F_{i, 6}+R_{i} \Omega_{i}
$$

with $\Omega=\left(g_{i}^{1}, g_{i}^{2} \ldots, g_{i}^{\prime \prime}\right)^{T}$. Here $\operatorname{diag}\left(z^{\prime}\right)$, denotes a diagonal matrix with diagonal elements $\because$. Numerical experiments with the use of the two forms of the numerical fluxes $(4.4 \mathrm{~b})$ and (4.5a.b) together with $\psi(z)=|z|$ on a quasi-one-dimensional divergent nozzle problem show that there is no visible improvement in accuracy of one over the other. By inspection, the operations count of the numerical flux $(4.5 \mathrm{a}, \mathrm{b})$ is higher than $(4.4 \mathrm{~b})$. Therefore, $(4.4 \mathrm{~b})$ is favored over $(4.5 a, b)$.

\section{EXTENSION OF THE IMPLICIT SCHEME BY THE ALTERNATING DIRECTION IMPLICIT IADII METHOD}

In order to solve for $U^{n-1}$ in (4.4a), one needs to solve a set of nonlinear algebraic equations. For computational efficiency, consider the following solution strategies. First. linearize the implicit operator in several ways as in the scalar case. Then construct an alternating direction implicit (ADI) form for the linearized implicit algorithms. The final step is to either use the $\mathrm{ADI}$ form as the solution algorithm, or use the ADI form as the predictor step and the original nonlinear algorithm (4.4) as the corrector step (with the numerical flux values evaluated at the predicted solution). The predictor-corrector method is proposed mainly because of its use (a) as a vehicle to compensate for the simplification of the various linearized implicit operators. and (b) for the possible improvement in convergence rate for steady-state applications.

Linearized nonconservative implicit ( $L N I)$ form

The corresponding LNI form (3.9) for the rwo-dimensional scheme (4.4) is

$$
\begin{aligned}
& {\left[I-\lambda^{\prime} \theta J_{1+12 . k}^{-} \Delta_{i-12}+\lambda^{\prime} \theta J_{i-1}^{-} \lambda_{k} \Delta_{i-1: 2}-\lambda^{\prime} \theta K_{i, k+1}^{-} \Delta_{h-1} 2\right.} \\
& \left.+\lambda \theta K_{j . h-12}^{+} \Delta_{k-1}\right]\left(U^{n-1}-U^{\prime \prime}\right)
\end{aligned}
$$

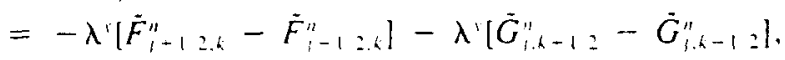

where

$$
\begin{aligned}
& J_{i+1}^{*} 2.4=\left(R_{1} \operatorname{diag}\left(C_{1}^{*}\right) R_{1}^{-1}\right)_{1-1}^{\prime \prime}, \ldots . \\
& K_{j, k+12}^{-}=\left(R_{y} \operatorname{diag}\left(C_{1}^{*}\right) R_{1}^{-1}\right)_{, k+12}^{n}
\end{aligned}
$$


and

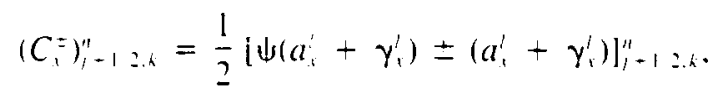

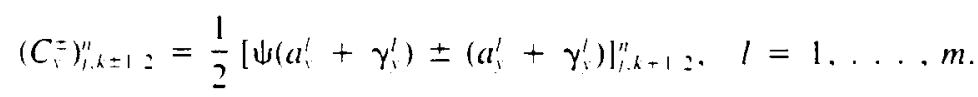

Here the operator $\Delta_{1+1}$, operating on $U$. means $\Delta_{i-1}, U=U_{1+1 . k}-U_{1 . k}$.

Notice that in each coordinate direction (5.1) is a spatially second-order-accurate, fivepoint scheme, yet the iteration matrix associated with (5.1) in that direction is block tridiagonal. Normally, the matrix associated with a five-point stencil scheme would have been a block pentadiagonal matrix.

To calculate (5.lb.c) at every time step is quite costly. For steady-state applications, (5.1) can be simplified even more by setting $\gamma=0$ in (5.2) since it is only necessary for the scheme to be second order after it reaches steady state. The time integration and the entire implicit operator can be viewed as a relaxation procedure for the steady-state solution.

A numerical experiment for a one-dimensional gas dynamics problem shows that the LNI form with $y \neq 0$ or $\gamma=0$ on the left-hand side has a fairly rapid convergence rate and gives good shock resolution. However. numerical experiments with an ADI form of (5.1)[4] show that the LNI form does not have a good convergence rate. One possible way of solving (5.1) is by a different type of relaxation method. This will be the subject of a future investigation. For the rest of the paper. the LNI approach will be abandoned and in favor of the linearized conservative implicit form (3.13).

\section{Linearized conservative implicit $(L C I)$ form}

The $\mathrm{LCl}$ form corresponding to (3.13) for the two-dimensional scheme (4.4) is

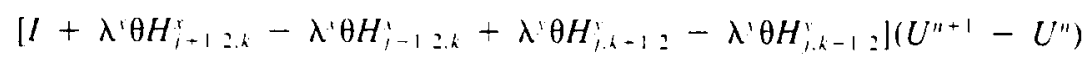

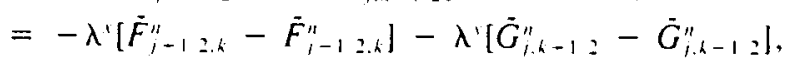

where

$$
\begin{aligned}
& H_{i-12 . k}^{r}=\frac{1}{2}\left[A_{j+1 . k}+\Omega_{i+12 . k}^{r}\right]^{\prime \prime} \\
& H_{, k-12=}^{i}=\frac{1}{2}\left[B_{i, k+1}+\Omega_{j, k+1}^{r}\right]^{\prime \prime},
\end{aligned}
$$

with $A$ and $B$ equal to the Jacobian of the fluxes $F$ and $G$. and

$$
\begin{aligned}
& \Omega_{j+1, k}^{i}=\left(R_{1} \operatorname{diag}\left[\beta^{l}-\psi\left(a^{l}+\gamma^{\prime}\right) \mid R_{r}^{-1}\right)_{i+1, \lambda_{i+1},},\right. \\
& \Omega_{j, k-1:}^{\prime}=\left(R_{y} \operatorname{diag}\left[\beta^{\prime}-\psi\left(a^{\prime}+\gamma^{\prime}\right) \mid R_{1}^{-1}\right)_{k+1} \Delta_{k-1}=\right.
\end{aligned}
$$

where

$$
\beta_{i+12}^{l}=\frac{\left(g_{l}^{\prime}+g_{1-1}^{\prime}\right)}{\alpha_{1+12}^{\prime}} ; \quad \beta_{k+12}^{\prime}=\frac{\left(g_{k}^{l}+g_{k+1}^{\prime}\right)}{\alpha_{k+12}^{\prime}} .
$$

The nonstandard notation

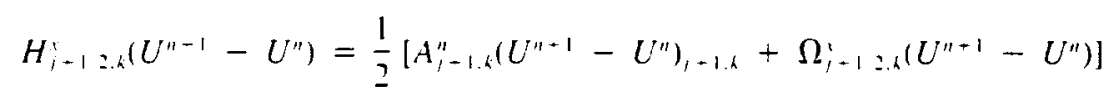

is used and the difference operator $\Omega_{;-1,2 .}$ is defined in (5.4a). 
For steady-state application, one way to simplify $(5.4)$ is to use a spatially first-order implicit operator; i.e. by redefining $(5.4 a)$ and $(5.4 b)$ as

$$
\begin{aligned}
& \left.\Omega_{j+12, k}^{\mathrm{r}}=\left(R_{\mathrm{r}} \operatorname{diag}\left[-\psi\left(a^{i}\right)\right] R_{\mathrm{r}}^{+1}\right)_{t-1,2}\right]_{,-1}, \\
& \Omega_{j, k+1,2}^{v}=\left(R_{y} \operatorname{diag}\left[-\psi\left(a^{i}\right)\right] R_{r}^{-1}\right)_{k-1} \nu_{i-1} \text {. }
\end{aligned}
$$

The computation can be reduced even more if $\Omega_{,-1, k}^{x}$ and $\Omega_{j, k-1}$ are simplified to diagonal matrices. For example, redefine (5.5) as

$$
\begin{aligned}
& \Omega_{j+1,2 . k}^{r}=\left(\operatorname{diag}\left[-\max _{l} \psi\left(a^{\prime}\right)\right]\right)_{j+1: 2} \Delta_{j+12}, \\
& \Omega_{j, k+1: 2}^{r}=\left(\operatorname{diag}\left[-\max _{l} \psi\left(a^{\prime}\right)\right]\right)_{k+1 ; 2} \Delta_{k+1: .} .
\end{aligned}
$$

From here on, algorithm (5.3) together with (5.6) is referred to as the linearized conservative diagonal form. It turns out that this linearized conservative diagonal form is quite an attractive method for steady-state applications.

ADI form

Even with the above simplifications, it is still very costly to solve the two-dimensional difference equation (5.3). An ADI form of (5.3) will be adopted as

$$
\begin{aligned}
& {\left[I+\lambda^{r} \theta H_{j+1 / 2, k}^{r}-\lambda^{r} \theta H_{j-1 / 2, k}^{x}\right] D^{*}} \\
& =-\lambda^{r}\left[\bar{F}_{j-12, k}^{n}-\bar{F}_{j-1 ; 2, k}^{n}\right]-\lambda^{\prime}\left[\dot{G}_{j, k+1}^{n}=-\tilde{G}_{j, k-1}^{n}\right] . \\
& {\left[I+\lambda^{v} \theta H_{j, k+1}^{v} \geq-\lambda^{v} \theta H_{j, k-1,2}^{v}\right] D=D^{*} .} \\
& U^{n+1}=U^{n}+D \text {. }
\end{aligned}
$$

Observe that (5.7) is the original Beam and Warming[17] algorithm if $\Omega_{j+12 . k}^{r}=$ $\Omega_{j, k+1 ; 2}^{*}=0$ in $(5.3 \mathrm{~b}, \mathrm{c})$ and $\Phi_{j+1 / 2} R_{j+1: 2}$ in $(4.4 \mathrm{~b})$ is replaced by the conventional fourth-order dissipation term. The implementation of this ADI scheme into an existing central difference code (such as the code based on the Beam and Warming algorithm) is relatively simple. All one has to do is add the extra matrices $\Omega_{j-1,2 . k}^{x}$ and $\Omega_{j, k+1,2}$ for the implicit operator and a more sophisticated dissipation term $\Phi_{j+12} R_{j+1}(4.4 \mathrm{~b})$ for the explicit operator. For the case of the linearized conservative diagonal form (5.3) together with (5.6), no extra work is involved on the implicit operator, since $\Omega^{x}$ and $\Omega^{y}$ in (5.6) are diagonal matrices with equal elements, and can be saved while computing for the right-hand side.

From numerical experiments with the NACA0012 airfoil steady-state calculations, the linearized conservative diagonal $\mathrm{ADI}$ form is the most efficient scheme among the various proposed linearized methods for the case of $\theta=1$. No comparison has been made for timeaccurate calculations or for any other values of $\theta$. A study on the predictor-corrector step method (as mentioned at the beginning of the section) does not show a drastic change in convergence rate for the same airfoil calculations. In the next section. some numerical results for the linearized conservative ADI algorithm with $\theta=1$ (the backward Euler time differencing) will be shown.

\section{NUMERICAL RESLLTS FOR THE ELLER EQUATIONS}

The results presented here utilized a coordinate transformation transforming a general curvilinear physical space into a rectangle with uniform spacing of unit length. For the airfoil calculations, the actual geometry is mapped onto the computational rectangle such that all the boundary surfaces are edges of the rectangle. Figure 1 shows the transformation for a " $\mathrm{C}$ " mesh topology where a branch cut (wake cut) is used at the trailing edge of the airfoil. In " $\mathrm{O}$ " mesh topologies the wake cut boundary is periodic and slightly more computation is involved for the block tridiagonal inversion.

The generalized coordinates formulation of the algorithm, treatment of boundary conditions, 


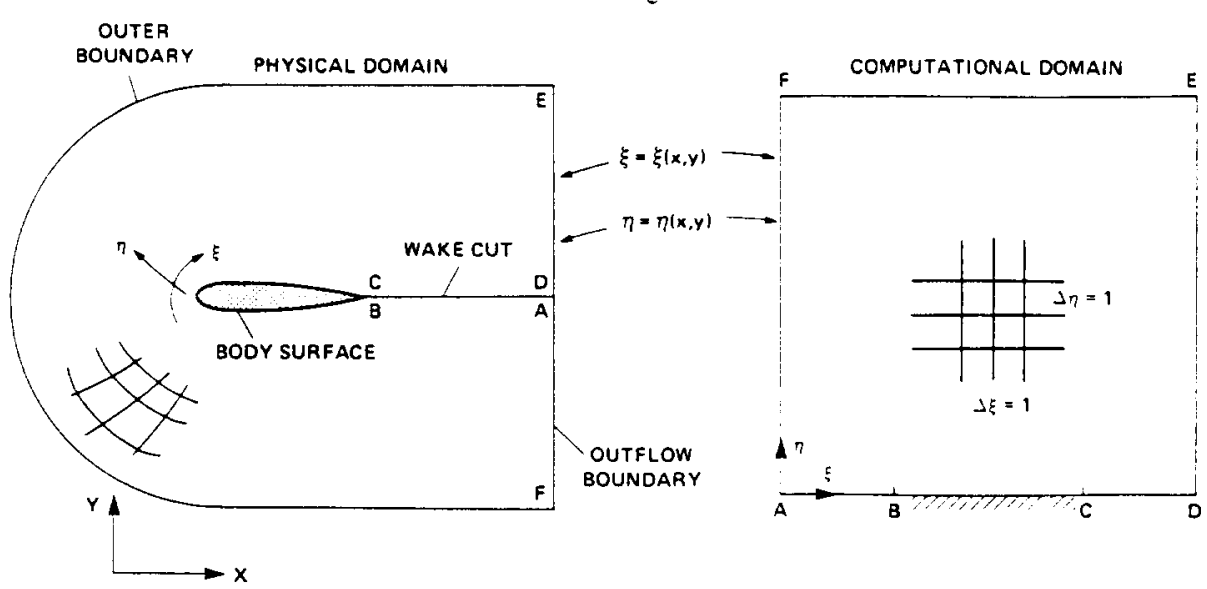

Fig. 1. Generalized coordinate transformation with " $\mathrm{C}$ " grid topology for airfoil calculations.

and more detailed description of the applications to the Euler equations of gas dynamics are described in [15]. Here some of the numerical results are shown to illustrate the accuracy and efficiency of the method for $\theta=1$.

Since the actual grids have widely varying cell sizes, a space-varying $\Delta t$ similar to the one used by Pulliam and Steger[18] is used as a vehicle to improve the convergence rate. The $\epsilon$ in $(2.7)$ is set to 0.125 for all cases. Numerical experiments show that the solutions are insensitive to the value of $\epsilon$ between 0.06 to 0.25 . A more sophisticated $\epsilon$ has been proposed in [8]. Computational experiments again show no visible improvement in efficiency or accuracy in using the more sophisticated formula while more computations are required. No artificial compression term as discussed in [4] is needed in all of the airfoil calculations.

The inviscid cases considered here are the NACA0012 airfoil with (a) $M_{*}=0.8, \alpha=$ 1.25, (b) $M_{x}=0.85, \alpha=1.00$, (c) $M_{x}=0.95, \alpha=0.0$. and (d) $M_{x}=1.2, \alpha=7.0$. Here $M_{\times}$is the freestream Mach number and $\alpha$ is the angle of attack. These are four of the cases which have been considered in the AGARD Fluid Dynamics Panel Working Group 07[5].

To show the accuracy of the scheme, a $249 \times 41 \mathrm{C}$ grid with no special clusterings on the upper or lower surface near the vicinity of the shocks is used. Figure 2 shows the grid distribution around the airfoil. The outer boundary is 24 chord lengths away from the body. Each case was initialized with a uniform freestream flow at the prescribed Mach number and angle of attack and used the same grid as shown in Fig. 2. Figures 3-6 show the pressure coefficients and Mach contours for all four cases. The symbol " + " on the pressure coefficient plots is used to indicate the computed values. The solid (upper surface) and the dashed (lower surface) lines are just connectors between grid points. The value $C_{P}^{*}$ in all of the pressure coefficient figures indicates the critical pressure coefficient. One can see in all cases that shocks can be captured within 1-2 grid points. When the same cases are run with the FLO52R code of Jameson[19] and the improved ARC2D code (version 150) of Pulliam and Steger[18], 3-4 points in the shock transition are generally observed. Away from the shocks, the three methods produce almost identical results. As a side remark, the accuracy and efficiency of FLO52R and the improved version of $\mathrm{ARC} 2 \mathrm{D}$ are comparable; see Ref. [18] for more details. Both codes use central difference in space with similar numerical dissipation terms but they use different time-stepping methods for steady-state applications. These two codes are widely circulated. Figures 7 and 8 show the comparison of the current scheme with ARC2D (version 150) for cases (a) and (b) using the same mesh as in Fig. 2. One can see that the current method captures the shock better than ARC2D. especially on the lower surface.

Here. as a guideline, the results of Pulliam and Barton[5] (using ARC2D, version 150) and the not-yet-published results of the AGARD Fluid Dynamics Panel Working Group 07 are used as the "exact" solutions. Pulliam and Barton computed all the cases with very fine grids of $561 \times 65$ and with very dense clustering near the shocks. Shock strengths and shock locations of the current calculations coincide very well with the fine-grid results of Pulliam and Barton. The present numerical experiments indicate that, in order to produce the same accuracy as the TVD scheme, special clustering of grid points near the shocks and/or denser grid has to be 


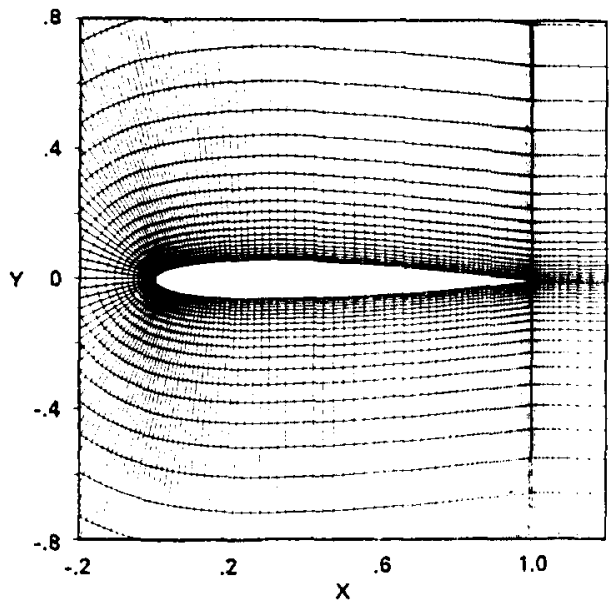

Fig. 2. The $249 \times 41 \mathrm{C}$ grid for the NACA0012 airfoil
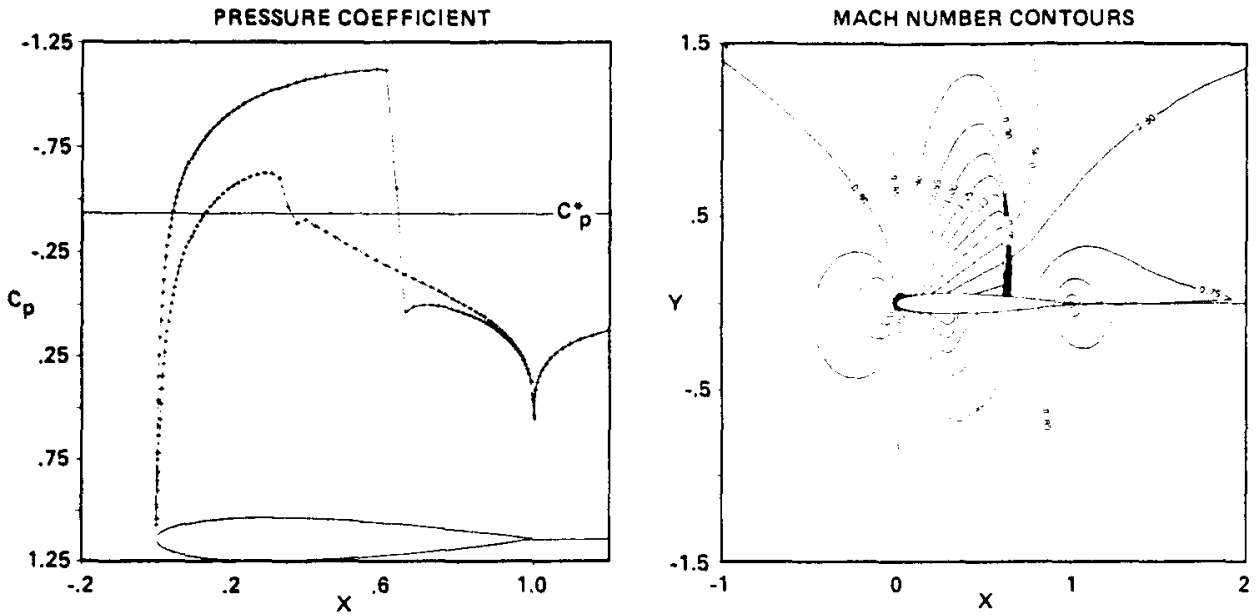

Fig. 3. Pressure coefficient and Mach contours for the NACA0012 airfoil with $M_{\iota}=0.8, \alpha=1.25$.
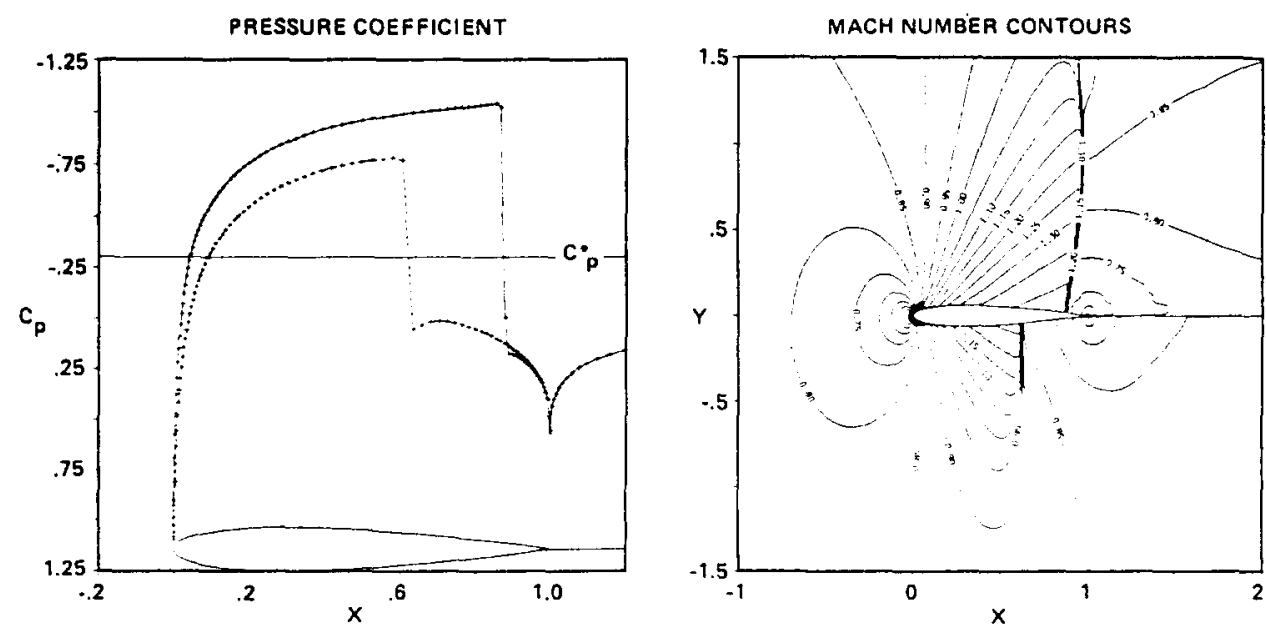

Fig. 4. Pressure coefficient and Mach contours for the NACA0012 airtoil with $M_{c}=0.85 . \alpha=1.0$. 

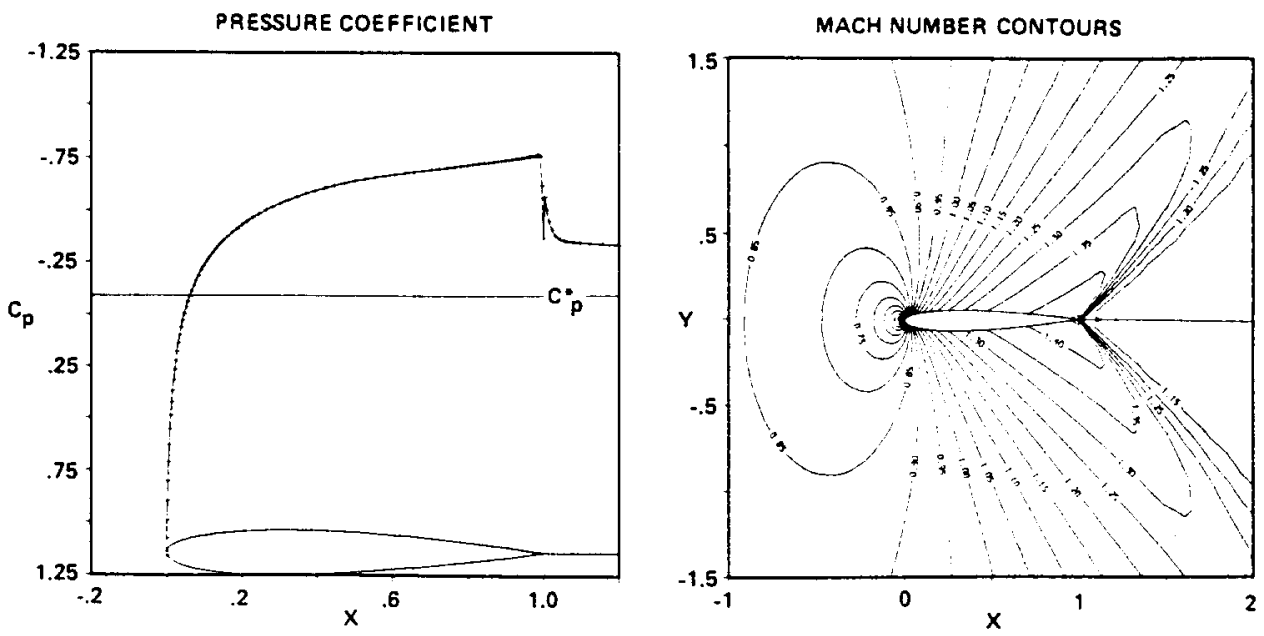

Fig. 5. Pressure coefficient and Mach contours for the NACA00I2 airtoil with $M=10.95, \alpha=0.0$
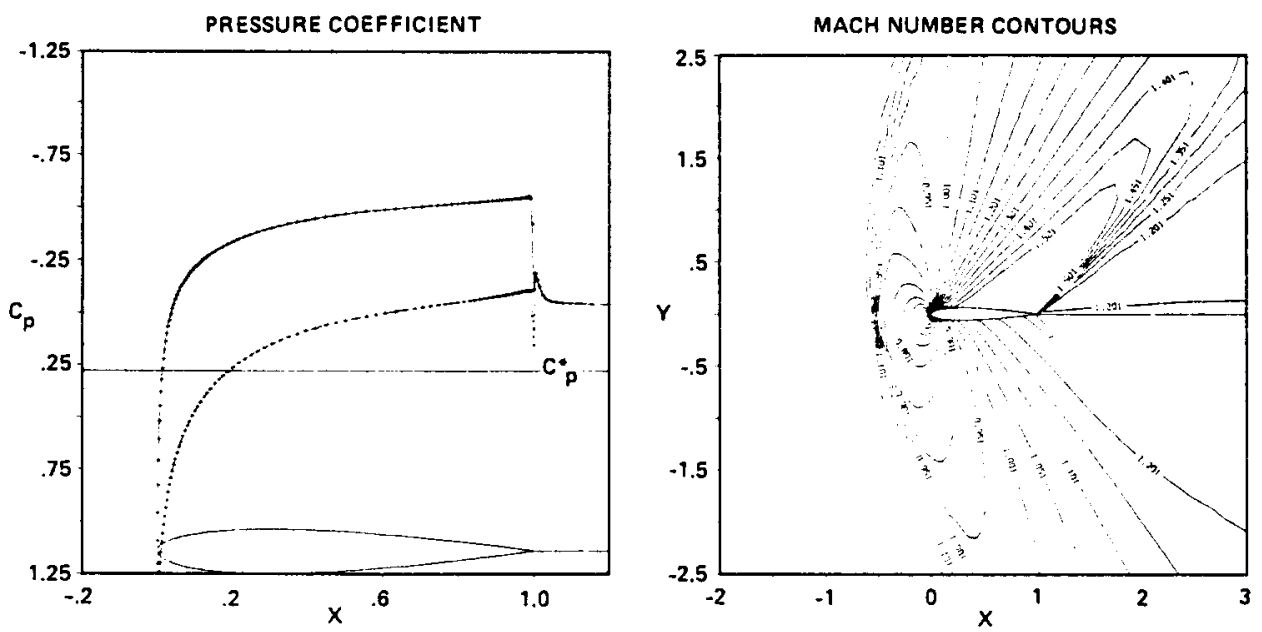

Fig. 6. Pressure coefficient and Mach contours for the NACA0012 airfoil with $h_{\alpha}=1.2 . \alpha=7.0$.

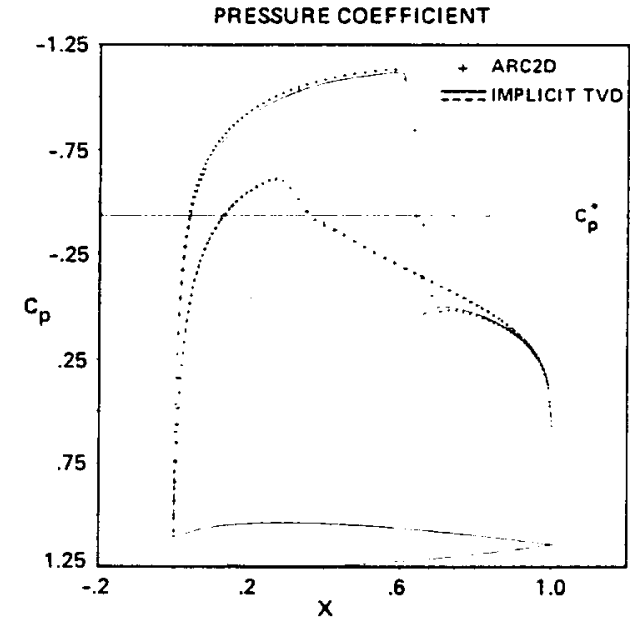

Fig. 7. Comparison of the current scheme with ARC2D for the NACA0012 airfoil with $M_{c}=0.8 . \alpha=1.25$. 


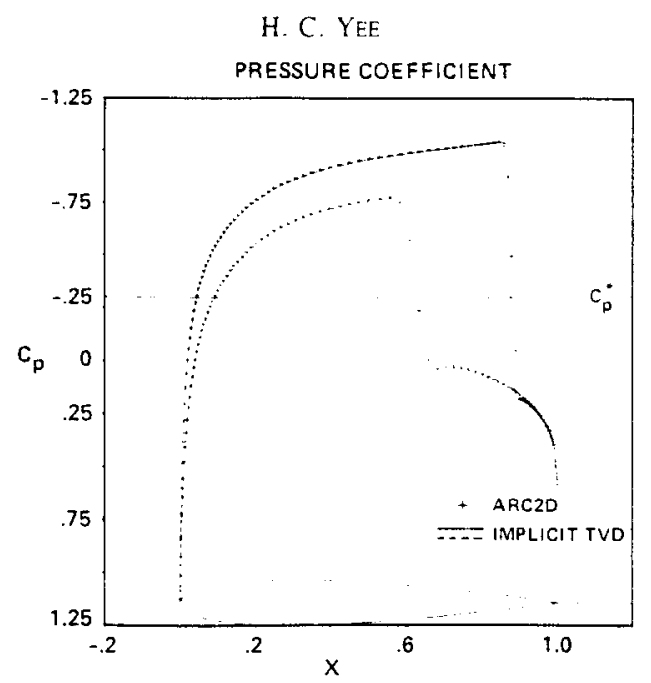

Fig. 8. Comparison of the current scheme with ARC2D for the NACA0012 airfoil with $u_{x}=0.85, \alpha=1.0$.

employed by FLO52R and ARC2D. Thus, the advantage of the current scheme over FLO52R and ARC2D is that the unfavorable condition in computations which arises from the high aspect ratio of grid spacing can be avoided. Moreover, the present method requires less storage and lower operation count than other TVD schemes[3].

For cases (a) and (a), the $L_{2}$-norm residual (of the explicit operator) reaches machine zero at around 3000 steps. A residual of $10^{-7}$ can be reached in around 800 steps. Cases (b) and (c) are slightly more difficult. The shocks appear to be oblique and not aligned with the $\mathrm{C}$ grid coordinate system. The convergence rates are slower. Since the computer code is not fully vectorized and is not coded in an optimized fashion. it requires $0.53 \mathrm{sec}$ per time step on the Cray XMP at the NASA Ames Research Center [based on the $(249 \times 41) \mathrm{C}$ grid]. A careful recoding could possibly increase the speed by a factor of 2 or more. When the vectorized option is turned off, the current code requires $1.69 \mathrm{sec}$ per time step, while ARC2D with the diagonal form[18] requires $1.01 \mathrm{sec}$ per time step. This indicates that the present method requires $68 \%$ more computation time than ARC2D.

The form of the modified flux function

In all of the inviscid calculations, the linearized conservative diagonal form (5.3) together with (5.6) is used, with a slightly simplified form of the modified flux with

$$
g_{j}^{l}+g_{j+1}^{l}=\sigma_{j-12}^{\prime}\left(\bar{g}_{l}^{\prime}+\bar{g}_{,-1}^{l}\right)
$$

and

$$
\tilde{g}_{j}^{l}=S \cdot \max \left[0, \min \left(\left|\alpha_{j+1}^{\prime}\right| \cdot S \cdot \alpha_{j-12}^{\prime}\right)\right],
$$

where $\sigma_{j+1 / 2}^{l}, S$, and $\alpha_{j+1,2}^{l}$ are defined as before.

Figure 9 shows the pressure coefficient with $\left(g_{l}^{l}+g_{j-1}^{l}\right)$ defined in $(6.1$ a) compared with the ones defined in Eqs. (4.4c,d). Solid and dashed lines are numerical results using (4.4c.d), and + 's are numerical results using Eq. (6.1). It is found that a definite improvement in accuracy can be obtained by using (6.1). The definition of $g_{\text {, }}$ 's in $(6.1)$ is identical to $(4.4 c, d)$ in the constant-coefficient cases. As a side remark, there is no visible difference in accuracy hetween $(4.4 c, d)$ and $(6.1)$ for one-dimensional applications.

Evaluation of the symmetric averages $\mathrm{U}_{j+1} \ldots \mathrm{k}$ and $\mathrm{L}_{j, \mathrm{~h}-1}$

For a perfect gas, numerical experiments have been performed with two types of averaging for $U_{j-1,2 . k}$ and $U_{, k+1,2}$. The simplest form of $U_{1-1,2 . k}$ is the arithmetic average $U_{1+1, k}=$ $0.5\left(U_{j+1 . k}+U_{j, k}\right)$. The other. Roe's averaging[7]. is only applicable to a perfect gas. It has the computational advantage of perfectly resolving stationary discontinuities. For detailed implementation, see Ref. [4]. However, under certain conditions, such as highly irregular grids 
PRESSURE COEFFICIENT

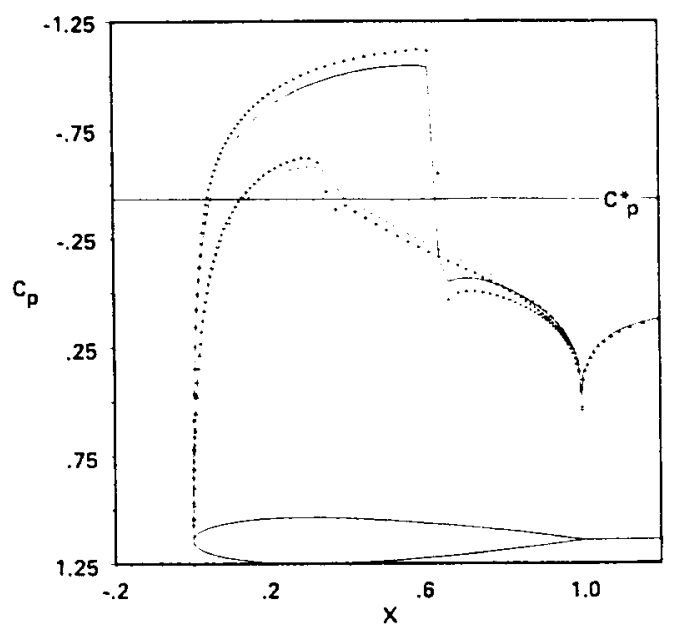

Fig. 9. Comparison of pressure coefficients between Egs. $(6.1)(+)$ and $4.4 \mathrm{c} . \mathrm{d})(-$ and $+\cdots)$ for the NACA0012 airfoil with $M_{x}=0.8$ and $\alpha=1.25$

or special flow conditions, the characteristic speeds $a_{j+1 / 2}^{\prime}$ can lie outside the interval $\left(a_{j}, a_{j-1}\right)$. Consequently, under this situation the direction of upwinding, which is determined solely by the sign of the $a_{j+1 / 2}^{\prime}$ 's on Roe's schemes, might be the opposite of what is desired. This special property of the Roe's averaging was first observed by M. Vinokur of NASA Ames Research Center. Roe's averaging has been tested on a variety of one- and two-dimensional gas dynamics problems $[12,14-16]$, and no sign of ill condition or instability was encountered. Numerical experiments with these two averages show no visible difference in numerical solutions for the above airfoil test cases. However, Roe's average requires slightly more computation.

\section{APPLICATION TO THE NAVIER-STOKES EQLATIONS}

Consider the two-dimensional mixed hyperbolic-parabolic system of conservation laws

$$
\frac{\partial U}{\partial t}+\frac{\partial F(U)}{\partial x}+\frac{\partial G(U)}{\partial y}=\frac{\partial F_{v}\left(U, U_{x}, U_{v}\right)}{\partial x}+\frac{\partial G_{v}\left(U, U_{x}, U_{v}\right)}{\partial y}
$$

Here $U, F(U)$ and $G(U)$ are the same as in (4.1). The additional vectors $F_{v}$ and $G_{v}$ are vector functions of not only the components of $U$, but also of $U_{x}$ and $U_{y}$, where $U_{x}=\partial U / \partial x$ and $U_{y}=\partial U / \partial y$. The compressible Navier-Stokes equations have the form (7.1). For a nondimensional form of the Naiver-Stokes equations,

$$
F_{v}=\frac{1}{\operatorname{Re}}\left[\begin{array}{c}
0 \\
\tau_{r x} \\
\tau_{r y} \\
e_{x}
\end{array}\right], \quad G_{v}=\frac{1}{\operatorname{Re}}\left[\begin{array}{c}
0 \\
\tau_{r y} \\
\tau_{y y} \\
e_{y}
\end{array}\right],
$$

with

$$
\begin{aligned}
\tau_{x x} & =\mu\left(4 u_{x}-2 v_{y}\right) / 3, \\
\tau_{x y} & =\mu\left(u_{y}+v_{x}\right), \\
\tau_{y y} & =\mu\left(-2 u_{x}+4 v_{y}\right) / 3, \\
e_{x} & =u \tau_{x x}+v \tau_{x y}+\mu P r^{-1}(\gamma-1)^{-1} \frac{\partial c^{2}}{\partial x}, \\
e_{y} & =u \tau_{x y}+v \tau_{y y}+\mu \operatorname{Pr}^{-1}(\gamma-1)^{-1} \frac{\partial c^{2}}{\partial y},
\end{aligned}
$$


where $\gamma$ is the ratio of specific heats. The dynamic viscosity is $\mu$ and typically consists of a constant plus a computed turbulent eddy viscosity. Re and $\operatorname{Pr}$ are the Reynolds number and Prandtl number.

A thin-layer approximation of the Navier-Stokes equation is made by resolving the viscous terms in a thin layer near the body[20]. Viscous terms in $x$, which is the direction along the solid body, are neglected. and terms in y are retained. Equation (7.1) thus simplifies to

$$
\frac{\partial U}{\partial t}+\frac{\partial F(U)}{\partial x}+\frac{\partial G(U)}{\partial y}=\frac{\partial G_{1}\left(C_{2} C_{1} \cdot U_{1}\right)}{\partial y}
$$

In general, for complex configurations, one does not have sufficient computer power to resolve the full Navier-Stokes equation. For sufficiently high Reynolds number. the thin-layer NavierStokes equations prove to be a useful approximation in a variety of applications.

For steady-state application. a simple algorithm utilizing the TVD scheme for the NavierStokes equations is to difference the hyperbolic terms the same way as before. and then central difference the viscous term. The final algorithm is the same as Eqs. (5.7) except that the spatial central differencing of the viscous term is added to the right-hand side of (5.7). It will be shown later that this simple-minded algorithm produces a fairly good solution for the case of a RAE 2822 airfoil calculation.

For time-accurate calculations, time accuracy is just as important as spatial accuracy for both the implicit and the explicit operator, and it is not apparent how to extend the current implicit method efficiently with the viscous term included. One way is to use a different time differencing, such as the Runge-Kutta method. A different approach is proposed here.

The unsteady compressible Navier-Stokes equation with turbulence models generally lead to an extremely "stiff" nonlinear system. In the numerical solution of such problems, it is sometimes more advantageous to treat the terms responsible for the severe time-step restriction implicitly and handle the remaining terms explicitly. The approach proposed here is similar to that of MacCormack[21].

The proposed method is to time split the equation into a hyperbolic part and a parabolic part. The hyperbolic part is solved with an explicit second-order TVD scheme viat the locallyone-dimensional (LOD) time-splitting method of Strang type (a fractional-step approach as described in Ref. [4]). The parabolic part is solved with an ADI implicit method with central difference in space. For the thin-layer Navier-Stokes equations. there is only one viscous term to worry about. Therefore, the parabolic part is especially easy to solve with one simple matrix inversion.

Let $\angle{ }_{x}^{\prime}, \angle$ be the LOD split hyperbolic finite-difference operator in the $x$ and $y$ directions. Furthermore. let $l_{s v}$ be the split parabolic operator in the $y$ direction. Then a fractional step method for the thin-layer Navier-Stokes Eqs. (7.4) will look like

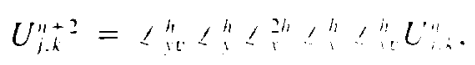

where $h=\Delta t$, and $Z_{r}^{h} U_{j, k}^{n}$, for example, is

$$
\left\langle{ }_{1}^{\prime \prime} U_{1 . k}^{\prime \prime}=U_{1 . k}^{n}-\lambda^{\prime}\left|\dot{F}_{1-12, k}-\dot{F}_{1-1, \ldots}\right| .\right.
$$

with $\tilde{F}_{j+1: 2 . k}$ defined in $(4.4 \mathrm{~b})$ and $\sigma(z)$ in $(3.7 \mathrm{a})$. The operator $\ldots$ which solves for the viscous (parabolic) term $G_{i}$. can be any one of the stable implicit schemes designed specially for parabolic equations (see, for example, MacCormack[21] and Beam and Warming[17]). Details and numerical experiments with this approach for transient calculations will appear in a separate article. Here we only illustrate some results for the first approach for steady-state calculations.

\section{NLMERICAL RESLLTS FOR THE VAVIER-STOKES EQLATIONS}

The viscous case considered here is the RAE2822 airfoil with $M=0.73 . \alpha=2.79$ and the Reynolds number $\mathrm{Re}=6.5 \times 10^{\mathrm{h}}$. The grid used is a $249 \times 51 \mathrm{O}$ grid [see Fig. $\left.\mid 0(a)\right]$. 


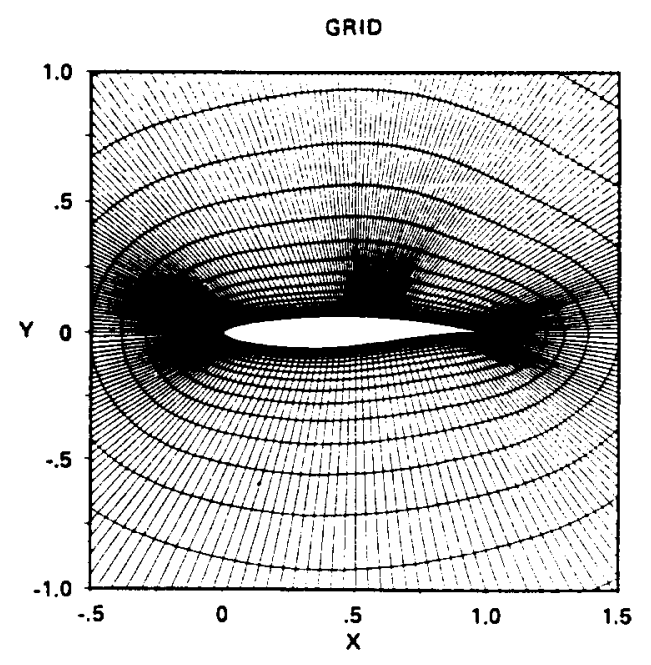

(a)

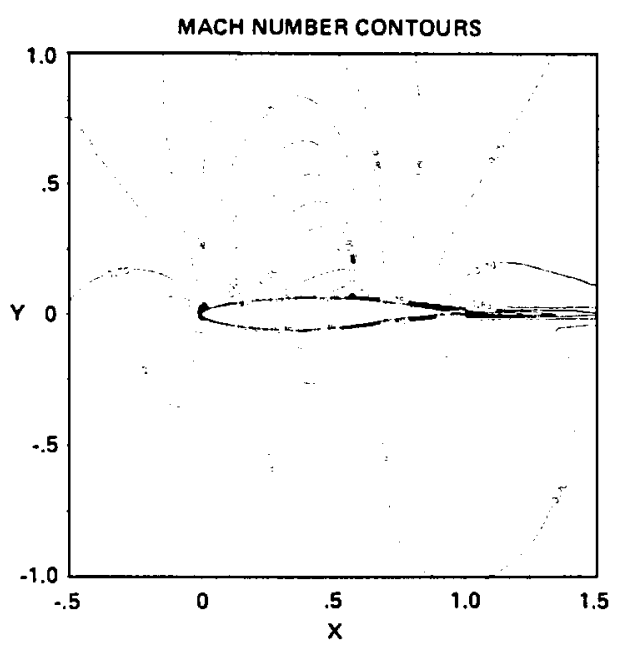

(c)

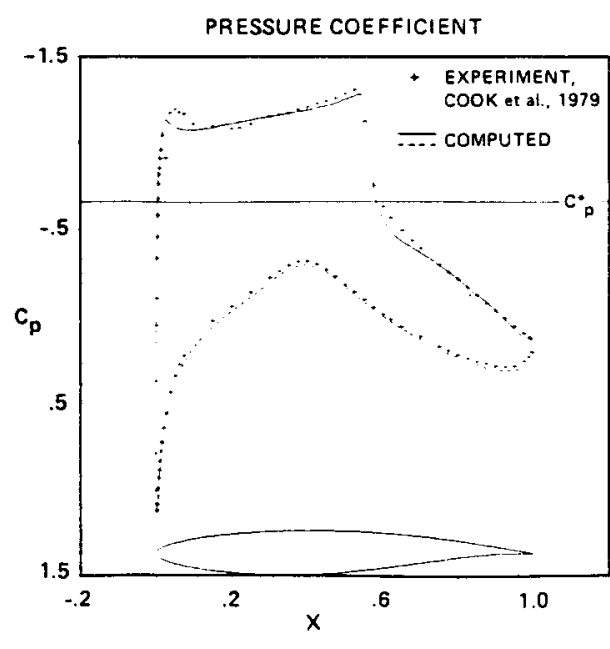

(b)

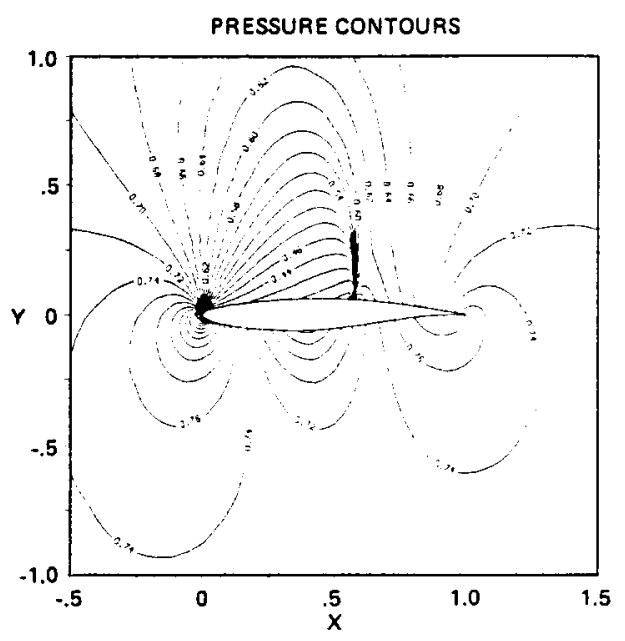

(d)

Fig. 10. The $249 \times 51^{\prime \prime} \mathrm{O}$ " grid, pressure coefficient, Mach contours, and pressure contours for the RAE2822 airfoil with $M_{\mathrm{x}}=0.73, \alpha=2.79, \mathrm{Re}=6.5 \times 10^{\circ}$

The thin-layer Navier-Stokes equations with the algebraic turbulence model of Baldwin and Lomax [20] are used and the transition is fixed at $3 \%$ of chord. Experimental data due to Cook et al. [22] are used for comparison. Figure 10(b) shows the pressure coefficients compared with experiment. The solid (upper-surface) and dashed (lower-surface) lines are the numerical solution for the present method. The + 's are the experimental data. The overall comparison with experiment is quite good. Figures $10(\mathrm{c}, \mathrm{d})$ show the Mach contours and pressure contours. The $L_{2}$-norm residual of $10^{-7}$ can be reached in around 900 steps. For this case, it requires approximately $1 \mathrm{sec}$ per time step. The extra CPU time required for the viscous case is mainly because of the additional computation due to the viscous term, the periodic boundary condition for the implicit operator, and the slightly bigger grid than in the inviscid case.

Again a space-varying $\Delta t$ similar to that of the inviscid case is used. The calculations shown in Fig. 10 use the expression (6.1) for $g$. The same difference method for the convection terms as the inviscid airfoil cases is used. The effect of the various turbulence models and the use of smoother transition operators as studied by Mehta et al.[23] have not been tried. They have found, in certain problems, that their proposed modification can improve convergence rate and accuracy. This will be a subject of a future investigation. 
Numerical experiments for the airfoil calculations show that the application of the proposed linearized second-order implicit TVD scheme generates good shock resolution for steady-state computations. The method is also applicable to viscous calculations. Very good agreement with experimental data was obtained for a case of the RAE2822 airfoil. The numerical dissipation is built in and has an automatic feedback mechanism. The current algorithm requires less storage and operation count than other TVD schemes[3]. The method is quite robust, fairly efficient, and can easily be implemented into an existing central difference code. In addition, the same formulation is also applicable to the three-dimensional Euler and Navier-Stokes equations.

Acknowledgment-The author wishes to thank Thomas Pulliam for his generous assistance on the airtoil geometries The permission to use his well-documented computer code " ARC2D" as a base code for the development of a research code for the present study is gratefully acknowledged

\section{REFERENCES}

1. A. Harten. A high resolution scheme for the computation of weak solutions of hyperbolic conservation laws. NYL Report. Oct., 1981: J. Comp. Phvs. 49, 357-393 (1983).

2. A. Harten, On a class of high resolution total-variation-stable finite-difference schemes. NYL Report. Oct., 1982 SIAM J. Numer. Anal. 21. 1-23 (1984).

3. H. C. Yee, On the implementation of a class of upwind schemes for system of hyperbolic conservation law. NASA Technical Memorandum 86839 (Sept. 1985).

4. H. C. Yee. R. F. Warming and A. Harten, Implicit total variation diminishing (TVD) schemes for steady-state calculations. AlAA Paper No. 83-1902. Proc. of the AIAA 6th Computational Fluid Dynamics Conference. Danvers. Mass., July. 1983; also in J. Comp. Phys. 57. 327-360 (1985).

5. T. H. Pulliam and J. T. Barton. Euler computations of AGARD working group 07 airfoil test cases. AlAA Paper No. $85-0018(1985)$

6. L. C. Huang, Psuedo-unsteady difference schemes for discontintuous solutions of steady-state, one-dimensional fluid dynamics problems. J. Comp. Phvs. 42. 195-211 (1981).

7. P. L. Roe, Approximate Riemann solvers, parameter vectors, and difference schemes. J. Comp. Phvs. 43, 357$372(1981)$.

8. A. Harten and J. M. Hyman, A self-adjusting grid for the computation of weak solutions of hyperbolic conservation laws. J. Comp. Phis. 50, 235-269 (1983)

9. P. K. Sweby. High resolution schemes using flux limiters for hyperbolic conservation laws. S/A.H J. Num. Analy. 21. $995-1011(1984)$

10. P. L. Roe. Some contributions to the modelling of discontinuous flows, in Proceedings of the A.WS-S/AM Summer Seminar on Large-Scale Computation in Fluid Mechanics. June 27-Jull. 8. 1983, Lectures in Applied Mathematics Vol. 22. AMS (1985).

11. A. Harten and S. Osher, Uniformly high order nonoscillatory schemes. UCLA Math. Report (1985).

12. H. C. Yee. R. F. Warming and A. Harten. Application of TVD schemes for the Euler equations of gas dynamics. in Proc. of the AMS-SIA.M Summer Seminar on Large-Scale Computation in Fluid Mechanics. June 27-July 8. 1983. Lectures in Applied Mathematics Vol. 22. AMS (1985).

13. J. B. Goodman and R. J. LeVeque. On the accuracy of stable schemes for 2D scalar conservation laws. NYL Report. New York (May [983).

14. H. C. Yee and P. Kutler. Application of second-order-accurate total variation diminishing (TVD) schemes to the Euler equations in general geometries. NASA TM-85845 (August 1983).

15. H. C. Yee and A. Harten. Implicit TVD schemes for hyperbolic conservation laws in curvilinear coordinates. AlAA Paper No. 85-1513-CP. Proc. of the AlAA 7th Computational Fluid Dinamics Conference. Cinn. Ohio. July 15-17, 1985. AIAA (1985)

16. H. C. Yee. On Symmetric and Lpwind TVD Schemes. Proceedings of the 6th GAMM Conference on Numerical Methods in Fluid Mechanics. (Sept. 25-27. 1985); also NASA Technical Memorandum 8684? (Sept. 1985).

17. R. Beam and R. F. Warming. An implicit finite-difference algorithm for hyperbolic systems in conservation law form. J. Comp. Phys. 22. 87-110 (1976).

18. T. H. Pulliam and J. Steger. Recent improvements in efficiency. accuracy and convergence for implicit approximate factorization algorithms. AIAA Paper No. 85.0360 (1985)

19. A. Jameson et al., Numerical solutions of the Euler equations by finite volume methods using Runge-Kutta timestepping schemes. AlAA Paper o. 81-1259 (1981).

20. B. Baldwin and H. Lomax. Thin layer approximation and algebraic model for separated turbulent flows. AlAt Paper No. $78-257$ (1978).

21. R. W. MacCormack, A rapid solver for hypervolic systems of equations. in Proceedings of the Fifth International Conference on Numerical Methods in Fluid Dvamics, Lecture Notes in Physics Vol. 59. Springer-Verlag (1976).

22. P. H. Cook et al. . Aerofoil RAE2822-Pressure distributions and boundary layer and wake measurements. AGARD AR- $138(1979)$.

23. Wehta et al., A Comparison of Interactive boundary layer and thin-layer Navier-Stokes procedures. in Proceeding of the 3rd Symposium on Numerical and Phisical Aspects of Aerodinamic Flons." Long Beach. Jan. 21-24. 1985. 